\title{
Size fluctuations of the initial source and the event-by-event transverse momentum fluctuations in relativistic heavy-ion collisions*
}

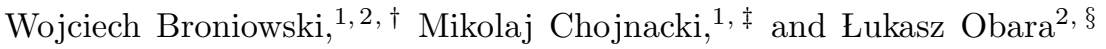 \\ ${ }^{1}$ The H. Niewodniczański Institute of Nuclear Physics, \\ Polish Academy of Sciences, PL-31342 Kraków, Poland \\ ${ }^{2}$ Institute of Physics, Jan Kochanowski University, PL-25406 Kielce, Poland
}

(Dated: ver. 2, 1 October 2009)

\begin{abstract}
We show that the event-by-event fluctuations of the transverse size of the initial source, which follow directly from the Glauber treatment of the earliest stage of relativistic heavy-ion collisions, cause, after hydrodynamic evolution, fluctuations of the transverse flow velocity at hadronic freezeout. This in turn leads to event-by-event fluctuations of the average transverse momentum, $\left\langle p_{T}\right\rangle$. Simulations with GLISSANDO for the Glauber phase, followed by a realistic hydrodynamic evolution and statistical hadronization carried with THERMINATOR, lead to agreement with the RHIC data. In particular, the magnitude of the effect, its centrality dependence, and the weak dependence on the incident energy are properly reproduced. Our results show that bulk of the observed event-by-event $\left\langle p_{T}\right\rangle$ fluctuations may be explained by the fluctuations of the size of the initial source.
\end{abstract}

PACS numbers: 25.75.-q, 25.75.Dw, 25.75.Ld

Keywords: relativistic heavy-ion collisions, transverse-momentum fluctuations, Glauber models, hydrodynamics, statistical hadronization, SPS, RHIC, LHC

We propose a new mechanism for generating the transverse-momentum fluctuations in relativistic heavyion collisions, based on the fluctuations of the initial size of the formed system and its subsequent hydrodynamic evolution. It is well established that a successful description of the physics of relativistic heavy-ion collisions is achieved with the help of relativistic hydrodynamics $[1,2,3,4,5,6,7,6,9,10,11,12]$, effective at proper times ranging typically from about a fraction of a $\mathrm{fm} / \mathrm{c}$ to a few $\mathrm{fm} / \mathrm{c}$, where statistical hadronization takes over. Numerous observables can be reproduced that way, such as the momentum spectra, elliptic flow, or the HBT correlation radii [11, 12], measuring the system's space-time extension. The initial condition for hydrodynamics is usually obtained from the Glauber approach, leading to the wounded-nucleon picture [13] (a wounded nucleon is a nucleon, that has collided inelastically at least once) or its variants [14, 15]. When the initial condition is obtained via Glauber Monte Carlo simulations, its shape fluctuates, simply reflecting the randomness in positions of the nucleons in the colliding nuclei.

In this paper we show that the event-by-event fluctuations of the initial size are substantial, even when we consider the class of events with a strictly fixed number of the wounded nucleons, $N_{w}$. The fluctuations are then carried over by hydrodynamics to the fluctuations of the transverse flow velocity at the hadronic freeze-out, which in turn generate the event-by-event fluctuations of the average transverse momentum, $p_{T}$,

* Supported in part by the Polish Ministry of Science and Higher Education, grants N202 034 32/0918 and N202 249235.

${ }^{\dagger}$ Electronic address: Wojciech.Broniowski@ifj.edu.pl

${ }^{\ddagger}$ Electronic address: Mikolaj.Chojnacki@ifj.edu.pl

$\S$ Electronic address: lukaszo4@onet.eu
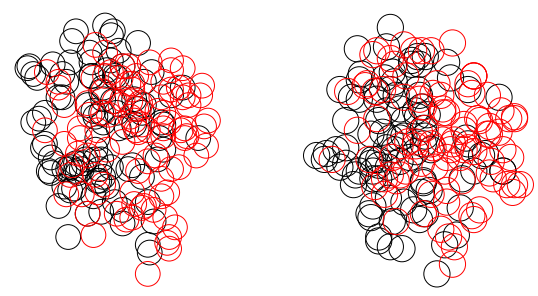

FIG. 1: (Color online) Two sample collisions, both with equal number of wounded nucleons, $N_{w}=198$, displayed as circles. The left and right cases have $\langle r\rangle=2.95 \mathrm{fm}$ and $2.83 \mathrm{fm}$, respectively.

of the produced hadrons. The mechanism is very simple: a more squeezed initial condition leads to faster expansion, larger flow, and, consequently, higher $\left\langle p_{T}\right\rangle$, while, on the conrary, a more stretched initial condition leads to slower expansion, lower flow, and lower $\left\langle p_{T}\right\rangle$. The event-by-event $p_{T}$ fluctuations have been a subject of intense theoretical and experimental studies [16, 17, 18, 19, 20, 21, 22, 23, 24, 25, 26, 27, 28, 29, 30], as they may reveal important details of the dynamics of the system. Effects of inhomogeneities for various observables have been studied in Ref. [31]. The event-byevent fluctuations of the initial shape have been studied in detail for its elliptic component, where they lead to enhanced elliptic flow [32, 33, 34, 35, 36, 37, 38, 39, 40, 41]. Our present study is similar in spirit, but it focuses on the size fluctuations.

Consider the average transverse size, defined in each event as (we use the wounded nucleon model for the simplicity of notation)

$$
\langle r\rangle=\sum_{i=1}^{N_{w}} \sqrt{x_{i}^{2}+y_{i}^{2}},
$$


where $x_{i}$ and $y_{i}$ are coordinates of a wounded nucleon in the transverse plane. Examples of the spatial distributions of wounded nucleons are shown if Fig. 1, where collisions of two ${ }^{197} \mathrm{Au}$ nuclei are viewed along the beam. We note that the two cases displayed in Fig. 1, although having equal numbers of wounded nucleons, have indeed a rather different shape and size. The original positions of nucleons in each nucleus are randomly generated from an appropriate Woods-Saxon distribution, with an additional constraint that the centers of nucleons in each nucleus cannot be closer than the expulsion radius $d=0.4 \mathrm{fm}$, which simulates the short-range repulsion. Nucleons from the two nuclei get wounded or undergo a binary collision when their centers pass closer to each other than the distance $d=\sqrt{\sigma_{N N} / \pi}$, where $\sigma_{N N}$ is the inelastic nucleon-nucleon cross section. For the highest SPS, RHIC, and LHC energies it is equal to 32,42 , and $63 \mathrm{mb}$, respectively.

We introduce the notation $\langle\langle\rangle$.$\rangle to indicate averaging$ over the events. In order to focus on the relative size of the effect we use the scaled standard deviation, defined for a fixed value of $N_{w}$ as $\sigma(\langle r\rangle) /\langle\langle r\rangle\rangle$. The results of our Monte Carlo simulations performed with GLISSANDO are shown in Fig. 2. We present the standard wounded nucleon model [13] (the three lower overlapping curves) and the mixed model [14, 15] (three upper curves), where the Glauber source distribution is formed from the fractions $(1-\alpha) / 2$ of the wounded nucleons and $\alpha$ of the binary collisions. The mixing parameter $\alpha$ is assumed to be equal to $0.12,0.145$, and 0.2 for the highest SPS, RHIC, and LHC energies, respectively. From the fact that the three curves for the wounded nucleon model overlap, we conclude that the effect is completely insensitive to the value of $\sigma_{N N}$ within the considered range. The observed moderate dependence on the energy for the mixed model originates entirely from the different values of the $\alpha$ parameter. For the binary collisions the size fluctuations are stronger than for the wounded nucleons, hence a larger value of $\alpha$ yield larger fluctuations. We note that the scaled variance of $\langle r\rangle$ is about $2.5-3.5 \%$ for central collisions, and grows towards the peripheral collisions approximately as $1 / \sqrt{N_{w}}$. Very similar results are obtained for other variants of Glauber models, in particular models with overlaid distribution of particles produced by each wounded nucleon [15]. We have also checked that using a Gaussian wounding profile $\sigma_{N N}(b)$ [42] for the $N N$ collisions, rather than the sharp wounding distance criterion applied here, leads to very similar curves. Furthermore, the use of the nucleon distributions including realistically the central $N N$ correlations, as given in Ref. [43], leads to practically no difference. In other words, the behavior displayed in Fig. 2 is robust, basically reflecting the statistical nature of the Glauber approach.

The next step, crucial in converting the size fluctuation into momentum fluctuations, is hydrodynamics. We use the perfect boost-invariant hydrodynamics described in detail in Ref. [1], which leads to a successful simultaneous description of the soft RHIC observ-

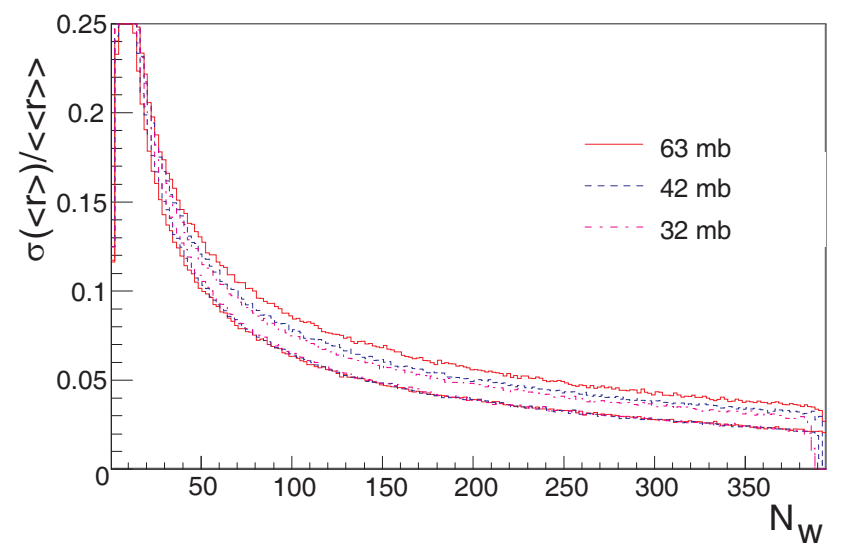

FIG. 2: (Color online) Event-by-event scaled standard deviation of the size parameter $\langle r\rangle$, evaluated at fixed values of the number of wounded nucleons, $N_{w}$. The three lower overlapping curves are for the wounded nucleon model at the NN cross section corresponding to the highest SPS (32 mb), RHIC (42 mb), and LHC (63 mb) energies. The three upper curves are for the mixed model at the subsequent energies. The dependence on the energy for the mixed model originates entirely from different values of the $\alpha$ mixing parameter, see the text for details.

TABLE I: Shape parameters $a$ and $b$, as well as the central temperature $T_{i}$, for various centrality classes for the highest RHIC energy of $\sqrt{s_{N N}}=200 \mathrm{GeV}$.

\begin{tabular}{r|rrrrrrrr}
\hline$c[\%]$ & $0-5$ & $5-10$ & $10-20$ & $20-30$ & $30-40$ & $40-50$ & $50-60$ & $60-70$ \\
\hline$a[\mathrm{fm}]$ & 2.70 & 2.54 & 2.38 & 2.00 & 1.77 & 1.58 & 1.40 & 1.22 \\
$b[\mathrm{fm}]$ & 2.93 & 2.85 & 2.74 & 2.59 & 2.45 & 2.31 & 2.16 & 2.02 \\
$T_{i}[\mathrm{MeV}]$ & 500 & 491 & 476 & 455 & 429 & 398 & 354 & 279 \\
\hline
\end{tabular}

ables at midrapidity, such as the $p_{T}$ spectra, elliptic flow, and the HBT radii, including the azimuthally-sensitive (azHBT) femtoscopy [44]. The essential ingredients of this approach are the Gaussian transverse initial condition, early start of the evolution $\left(\tau_{0}=0.25 \mathrm{fm} / \mathrm{c}\right)$, and the state-of-the art equation of state [11], incorporating a smooth crossover and interpolating between the lattice QCD results at high temperatures and the resonance gas at low temperatures. The initial transverse energy-density profile is assumed to have the simple form $\epsilon(x, y)=\epsilon_{0} \exp \left[-x^{2} /\left(2 a^{2}\right)-y^{2} /\left(2 b^{2}\right)\right]$, where $x$ and $y$ denote the transverse coordinates. The width parameters $a$ and $b$ depend on centrality. To obtain realistic values we run GLISSANDO [15] simulations, which include the eccentricity fluctuations. Then we match $a$ and $b$ to reproduce the values $\left\langle x^{2}\right\rangle$ and $\left\langle y^{2}\right\rangle$ from the GLISSANDO Monte Carlo profiles. Thus, by construction, the spatial rms radii of the initial condition and its eccentricity are the same as from the Glauber calculation. The values of the used width parameters are given in Table I. The central value of the initial temperature is adjusted in such a way that the multiplicity of produced particles is reproduced.

The average transverse momentum is also reproduced 
by our model, as can be seen from Fig. 3, where we compare the model predictions (solid line) to the STAR data [45], extrapolated to the full $p_{T}$ coverage. The dashed line corresponds to the model calculation with the STAR range $0.2 \mathrm{GeV}<p_{T}<2 \mathrm{GeV}$ used in Ref. [28].

Next, we analyze the hydrodynamic evolution with fluctuating initial conditions. Rather than doing an event-by-event calculation, which is tedious, it suffices (see the following) to see how much the predictions change when the $a$ and $b$ parameters are scaled by the values read off from Fig. 2. For instance, for $c=20-30 \%$, which corresponds to $N_{w} \simeq 165$, we note from Fig. 2 2 that the scaled standard deviation of the size fluctuations is $4.4 \%$ (for the wounded nucleon model). Thus we rescale $a$ and $b$ up and down by $4.4 \%$ and run the simulations. In addition, we also adjust the value of the central temperature $T_{i}$ in such a way, that the energy contained in the profile is preserved. This is natural, as the total energy deposited in the transverse plane should be (up to possible additional fluctuations) the same for a given number of elementary collisions. Hence a squeezed system has a higher central temperature than a stretched system. Thus, in some sense, we include also the temperature fluctuations discussed in Ref. [46]. Additional event-byevent energy fluctuations (in the considered pseudorapidity window $|\eta|<1$ ) could be added on top of the analyzed effect, which would act as another source of momentum fluctuations, not included in this work.

Hydrodynamics is run till the local temperature drops to $T_{f}=145 \mathrm{MeV}$ [11], where freezeout occurs. The freezeout hypersurfaces in the space of the transverse radius, $r$, and time, $t$, at $z=0$, as well as the transverse velocities, for the $5 \%$ squeezed and stretched cases for $c=20-30 \%$ are shown in Fig. 4. We note the following features: the maximum expansion velocities, in-

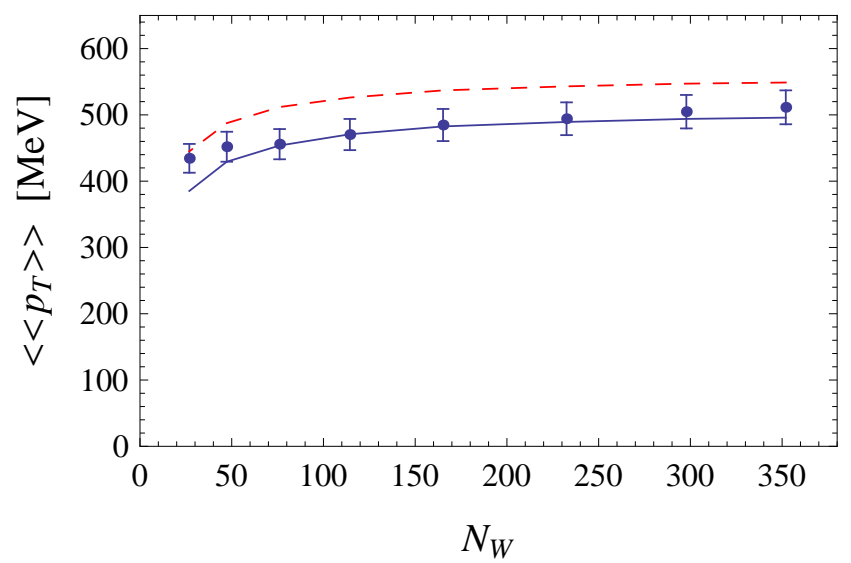

FIG. 3: (Color online) Dependence of the average transverse momentum, $\left\langle\left\langle p_{T}\right\rangle\right\rangle$, on the number of wounded nucleons. The solid (dashed) line corresponds to the model calculation in the full $p_{T}$ range (in the range of the STAR coverage $0.2 \mathrm{GeV}<p_{T}<2 \mathrm{GeV}$ ). The experimental points (obtained by extrapolating the $p_{T}$ distributions to the full range) are taken from Ref. [45].
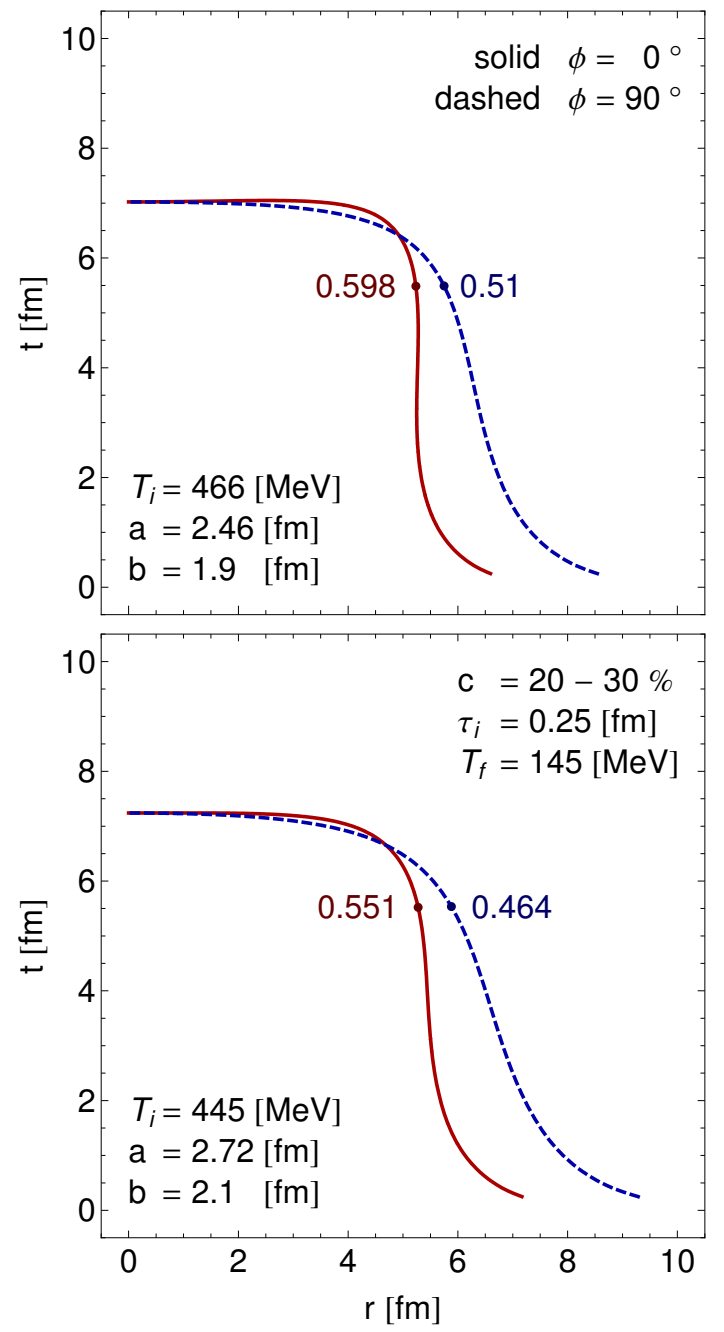

FIG. 4: (Color online) In-plane (solid) and out-of-plane (dashed) freezeout hypersurfaces in the transverse coordinatetime plane for $c=20-30 \%$, with the indicated values of the maximum transverse flow velocity in units of $c$. Top (bottom): the $5 \%$ squeezed (stretched) initial condition.

dicated by dots with labels, are about $10 \%$ larger for the squeezed case compared to the stretched case, in addition the squeezed case is somewhat more compact, i.e. the radius $r$ and the time $t$ at freezeout are slightly smaller.

The final stage of our calculation, turning the transverse-velocity fluctuations into the transversemomentum fluctuations, is the simulation of the statistical hadronization at freezeout with THERMINATOR [47], which includes all resonances and decay channels from SHARE [48]. According to the Cooper-Frye formalism, the particles (stable and unstable, which subsequently decay) are formed at the freezeout hypersurface according to appropriate statistical distributions. For the squeezed case they are more "pushed" with the larger flow velocity than for the stretched case, thus they acquire a higher average transverse momentum, $\left\langle\left\langle p_{T}\right\rangle\right\rangle$. The experimental cuts of the STAR detector are used, with $0.2 \mathrm{GeV}<p_{T}<2 \mathrm{GeV}$. 
In order to compare to the data, we analyze the STAR correlation measure [28], $\sigma_{\text {dyn }}^{2} \equiv\left\langle\Delta p_{i} \Delta p_{j}\right\rangle$. It can be shown to be equal to

$$
\sigma_{\mathrm{dyn}}^{2}=\sigma^{2}\left(\left\langle p_{T}\right\rangle\right)-\frac{1}{N_{\mathrm{ev}}} \sum_{k=1}^{N_{\mathrm{ev}}} \frac{\sigma_{k}^{2}\left(p_{T}\right)}{N_{k}},
$$

where $k$ is numbers the events, $\sigma_{k}^{2}\left(p_{T}\right)$ the variance of $p_{T}$ in a given event, and $N_{k}$ denotes the multiplicity of the event. By construction, the second term cancels the uncorrelated (purely statistical) fluctuations in the first term, leaving in $\sigma_{\text {dyn }}$ the dynamical correlations only. Our simulations do not include the statistical event-byevent fluctuations of $\left\langle p_{T}\right\rangle$, which would follow from the random nature of the statistical hadronization involving a finite number of particles. The procedure described below allows to avoid the tedious event-by-event studies in the extraction of $\sigma_{\text {dyn }}$ when it originates form the fluctuations of the initial condition.

The full statistical distribution $f\left(\left\langle p_{T}\right\rangle\right)$ in a given centrality class is a folding of the statistical distribution of $\left\langle p_{T}\right\rangle$ at a fixed initial size, centered around a certain $\bar{p}_{T}$, with the distribution of $\bar{p}_{T}$ centered around $\left\langle\left\langle p_{T}\right\rangle\right\rangle$. The value of $\bar{p}_{T}$, corresponding to a fixed initial size, fluctuates because of the fluctuations of the initial size. In the central regions both are close to gaussian distributions, hence we have to a very good approximation

$$
\begin{aligned}
& f\left(\left\langle p_{T}\right\rangle\right) \sim \int d^{2} \bar{p}_{T} \times \\
& \exp \left(-\frac{\left(\left\langle p_{T}\right\rangle-\bar{p}_{T}\right)^{2}}{2 \sigma_{\text {stat }}^{2}}\right) \exp \left(-\frac{\left(\bar{p}_{T}-\left\langle\left\langle p_{T}\right\rangle\right\rangle\right)^{2}}{2 \sigma_{\text {dyn }}^{2}}\right) .
\end{aligned}
$$

Carrying out the $\bar{p}_{T}$ integration yields the distribution of $\left\langle p_{T}\right\rangle$ centered around $\left\langle\left\langle p_{T}\right\rangle\right\rangle$ with the width parameter satisfying $\sigma^{2}=\sigma_{\text {stat }}^{2}+\sigma_{\text {dyn }}^{2}$. With the above factorized form we may obtain the second term directly from the distribution of the initial size parameter $\langle r\rangle$. Its distribution is also approximately gaussian,

$$
f(\langle r\rangle) \sim \exp \left(-\frac{(\langle r\rangle-\langle\langle r\rangle\rangle)^{2}}{2 \sigma^{2}(\langle r\rangle)}\right) .
$$

Because of the deterministic nature of hydrodynamics, $\bar{p}_{T}$ is a (complicated) function of $\langle r\rangle$. Nevertheless, in the vicinity of the central values we have from the Taylor expansion

$$
\bar{p}_{T}-\left\langle\left\langle p_{T}\right\rangle\right\rangle=\left.\frac{d \bar{p}_{T}}{d\langle r\rangle}\right|_{\langle r\rangle=\langle\langle r\rangle\rangle}(\langle r\rangle-\langle\langle r\rangle\rangle)+\ldots
$$

Substituting (5) into (4) and comparing to (3) we obtain the key result

$$
\sigma_{\mathrm{dyn}}=\left.\sigma(\langle r\rangle) \frac{d \bar{p}_{T}}{d\langle r\rangle}\right|_{\langle r\rangle=\langle\langle r\rangle\rangle},
$$

or for the scaled standard deviation

$$
\frac{\sigma_{\mathrm{dyn}}}{\left\langle\left\langle p_{T}\right\rangle\right\rangle}=\left.\frac{\sigma(\langle r\rangle)}{\langle\langle r\rangle\rangle} \frac{\langle\langle r\rangle\rangle}{\left\langle\left\langle p_{T}\right\rangle\right\rangle} \frac{d \bar{p}_{T}}{d\langle r\rangle}\right|_{\langle r\rangle=\langle\langle r\rangle\rangle} .
$$

This result bears similarity to the formula derived by Ollitrault [49], where $\sigma_{\text {dyn }} /\left\langle\left\langle p_{T}\right\rangle\right\rangle \sim \sigma(\langle s\rangle) /\langle\langle s\rangle\rangle$, with $s$ denoting the entropy density. The derivative in Eq. (7) can be computed numerically without difficulty by running two simulations at each centrality. We do it by comparing the average momenta obtained for the squeezed and stretched cases, as described above. Then

$$
\frac{\sigma_{\mathrm{dyn}}}{\left\langle\left\langle p_{T}\right\rangle\right\rangle}=\frac{\left\langle\left\langle p_{T}\right\rangle\right\rangle_{-}-\left\langle\left\langle p_{T}\right\rangle\right\rangle_{+}}{\left\langle\left\langle p_{T}\right\rangle\right\rangle_{-}+\left\langle\left\langle p_{T}\right\rangle\right\rangle_{+}}
$$

where - and + indicate the squeezed and stretched cases.

Our final result is shown in Fig. 5, where we compare the theoretical points to the experimental data from the STAR collaboration [28]. We note a strikingly good agreement between our calculation and the experiment, in particular for the standard wounded nucleon model. The mixed model, which is more realistic than the wounded-nucleon model, overshoots the data by about $20 \%$, producing even more fluctuations than needed. This may suggest that the coefficient $d \bar{p}_{T} /\left.d\langle r\rangle\right|_{\langle r\rangle=\langle\langle r\rangle\rangle}$ in Eq. (6) is somewhat too large. The value of this coefficient incorporates all the dynamics (the initial condition, hydrodynamics, statistical hadronization). Modifying these ingredients, not to mention the inclusion of viscosity effects, etc., may modify the value. Nevertheless, we note a proper magnitude of the effect and the correct dependence on centrality, with an approximate scaling $\sigma_{\mathrm{dyn}}\left(\left\langle p_{T}\right\rangle\right) /\left\langle\left\langle p_{T}\right\rangle\right\rangle \sim 1 / \sqrt{N_{w}}$. Also, since the results of Fig. 2 very weakly depend on $\sigma_{N N}$, to the extent that the hydrodynamic "pushing" is similar at various energies, our results should weakly depend on the incident energy, which is a desired experimental feature. We remark that the described mechanism works independently of the charge of particles. In order to describe the charge

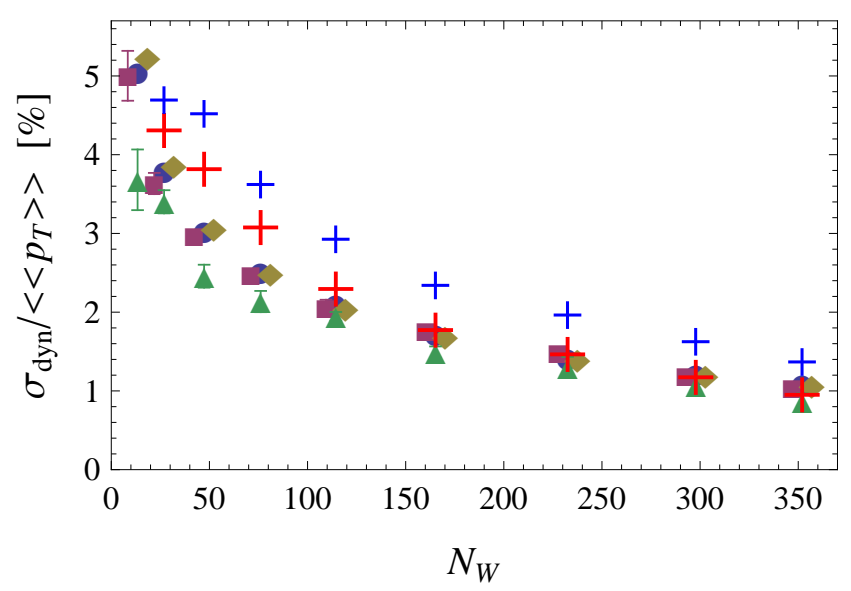

FIG. 5: (Color online) Comparison of the theoretical predictions for the scaled dynamical fluctuations, $\sigma_{\text {dyn }} /\left\langle\left\langle p_{T}\right\rangle\right\rangle$ (for $\left.\sqrt{s_{N N}}=200 \mathrm{GeV}\right)$, to the experimental data from the STAR collaboration [28]. The lower (upper) crosses indicate our results for the wounded nucleon model (mixed model). The experimental data range from $\sqrt{s_{N N}}=20 \mathrm{GeV}$ (triangles), through $130 \mathrm{GeV}$ (squares), $62 \mathrm{GeV}$ (diamonds), to $200 \mathrm{GeV}$ (dots). 
dependence of fluctuations (e.g. as observed in Ref. [25]) other mechanisms are necessary.

In conclusion, one can straightforwardly reproduce bulk of the dynamical event-by-event transverse momentum fluctuations, as measured at RHIC, with the mechanism based on fluctuations of the initial size, which are then carried over by hydrodynamics to the fluctuations of the transverse flow velocity, and consequently to the transverse momentum of the produced particles. The hydrodynamic pushing is crucial in this scheme. With a realistic hydrodynamics, which has been earlier used to uniformly describe the soft RHIC data, our analysis in- dicates that there may be little room for other, truly dynamical, sources of fluctuations, such as (mini)jets [27, 50] or the formation of clusters at freezeout [51, 52. Certainly, there are yet other sources of the $p_{T}$ correlations in addition to the afore-mentioned ones, such as the global momentum conservation, resonance decays, correlations from the elementary $N N$ collisions in the corona, however, these should be considered at the "background" mechanism of the size fluctuations described in this paper.

WB is grateful to Jean-Yves Ollitrault for pointing out Ref. [49].
[1] P. F. Kolb, J. Sollfrank, and U. W. Heinz, Phys. Rev. C62, 054909 (2000).

[2] S. A. Bass and A. Dumitru, Phys. Rev. C61, 064909 (2000).

[3] D. Teaney, J. Lauret, and E. V. Shuryak, Phys. Rev. Lett. 86, 4783 (2001).

[4] P. F. Kolb, U. W. Heinz, P. Huovinen, K. J. Eskola, and K. Tuominen, Nucl. Phys. A696, 197 (2001).

[5] T. Hirano and K. Tsuda, Phys. Rev. C66, 054905 (2002).

[6] P. Huovinen (2003), in Quark-Gluon Plasma 3, edited by R.C. Hwa and X.-N. Wang (World Scientific, Singapore, 2004), p. 600, nucl-th/0305064.

[7] P. F. Kolb and U. Heinz (2003), in Quark-Gluon Plasma 3, edited by R.C. Hwa and X.-N. Wang (World Scientific, Singapore, 2004), p. 634, nucl-th/0305084.

[8] E. V. Shuryak, Nucl. Phys. A750, 64 (2005).

[9] B. Muller, Acta Phys. Polon. B38, 3705 (2007).

[10] C. Nonaka, J. Phys. G34, S313 (2007).

[11] W. Broniowski, M. Chojnacki, W. Florkowski, and A. Kisiel, Phys. Rev. Lett. 101, 022301 (2008).

[12] S. Pratt, Acta Phys. Polon. B40, 1249 (2009).

[13] A. Bialas, M. Bleszynski, and W. Czyz, Nucl. Phys. B111, 461 (1976).

[14] D. Kharzeev and M. Nardi, Phys. Lett. B507, 121 (2001).

[15] W. Broniowski, M. Rybczynski, and P. Bozek, Comput. Phys. Commun. 180, 69 (2009).

[16] M. Gazdzicki and S. Mrowczynski, Z. Phys. C54, 127 (1992).

[17] L. Stodolsky, Phys. Rev. Lett. 75, 1044 (1995).

[18] E. V. Shuryak, Phys. Lett. B423, 9 (1998).

[19] S. Mrowczynski, Phys. Lett. B430, 9 (1998).

[20] S. A. Voloshin, V. Koch, and H. G. Ritter, Phys. Rev. C60, 024901 (1999).

[21] G. Baym and H. Heiselberg, Phys. Lett. B469, 7 (1999).

[22] S. A. Voloshin (STAR) (2001), nucl-ex/0109006.

[23] D. J. Prindle and T. A. Trainor (STAR), PoS CFRNC2006, 007 (2006).

[24] S. Mrowczynski, Acta Phys. Polon. B40, 1053 (2009).

[25] J. Adams et al. (STAR), Phys. Rev. C71, 064906 (2005).

[26] D. Adamova et al. (CERES), Nucl. Phys. A727, 97 (2003).

[27] S. S. Adler et al. (PHENIX), Phys. Rev. Lett. 93, 092301
(2004)

[28] J. Adams et al. (STAR), Phys. Rev. C72, 044902 (2005).

[29] K. Grebieszkow et al., PoS CPOD07, 022 (2007).

[30] T. Anticic et al. (NA49), Phys. Rev. C79, 044904 (2009).

[31] Y. Hama, R. P. G. Andrade, F. Grassi, W. L. Qian, and T. Kodama, Acta Phys. Polon. B40, 931 (2009).

[32] C. E. Aguiar, T. Kodama, T. Osada, and Y. Hama, J. Phys. G27, 75 (2001).

[33] M. Miller and R. Snellings (2003), nucl-ex/0312008.

[34] R. S. Bhalerao, J.-P. Blaizot, N. Borghini, and J.-Y. Ollitrault, Phys. Lett. B627, 49 (2005).

[35] R. Andrade, F. Grassi, Y. Hama, T. Kodama, and J. Socolowski, O., Phys. Rev. Lett. 97, 202302 (2006).

[36] S. A. Voloshin (2006), nucl-th/0606022.

[37] W. Broniowski, P. Bozek, and M. Rybczynski, Phys. Rev. C76, 054905 (2007).

[38] Y. Hama et al. (2007), 0711.4544.

[39] S. A. Voloshin, A. M. Poskanzer, A. Tang, and G. Wang (2007).

[40] S. Manly et al. (PHOBOS), Nucl. Phys. A774, 523 (2006).

[41] B. Alver et al. (PHOBOS) (2006), nucl-ex/0610037.

[42] A. Bialas and A. Bzdak, Phys. Lett. B649, 263 (2007).

[43] M. Alvioli, H. J. Drescher, and M. Strikman, Phys. Lett. B680, 225 (2009).

[44] A. Kisiel, W. Broniowski, M. Chojnacki, and W. Florkowski, Phys. Rev. C79, 014902 (2009).

[45] B. I. Abelev et al. (STAR), Phys. Rev. C79, 034909 (2009).

[46] R. Korus, S. Mrowczynski, M. Rybczynski, and Z. Wlodarczyk, Phys. Rev. C64, 054908 (2001).

[47] A. Kisiel, T. Taluc, W. Broniowski, and W. Florkowski, Comput. Phys. Commun. 174, 669 (2006).

[48] G. Torrieri et al., Comput. Phys. Commun. 167, 229 (2005).

[49] J.-Y. Ollitrault, Phys. Lett. B273, 32 (1991).

[50] Q.-j. Liu and T. A. Trainor, Phys. Lett. B567, 184 (2003).

[51] W. Broniowski, B. Hiller, W. Florkowski, and P. Bozek, Phys. Lett. B635, 290 (2006).

[52] B. Tomasik (2008), 0806.4770. 